\title{
Pacific
}

Journal of

Mathematics

\section{ON DISCRETE ISOMETRY GROUPS OF NEGATIVE} CURVATURE

GAVEn MARTiN 


\title{
ON DISCRETE ISOMETRY GROUPS OF NEGATIVE CURVATURE
}

\author{
Gaven J. Martin
}

\begin{abstract}
In this paper we extend well-known results concerning the algebraic limits and deformations of groups of hyperbolic isometries of hyperbolic 3-space, $\mathrm{H}^{3}$, to negatively curved groups. For us these will be groups of isometries of variable negative curvature metrics satisfying a pinching condition and in particular will include the $\mathbb{R}$-rank one Lie groups. We accomplish these goals, as in the hyperbolic case, by producing a version of Jørgensen's inequality for such groups. Using an appropriate normalisation we can consider algebraic limits and deformations of such groups in the homeomorphism group of the $n$-ball, $\operatorname{Hom}\left(B^{n}\right)$. We ask that the generators of each group move continuously or some sequence of generators have limits in $\operatorname{Hom}\left(\mathbf{B}^{n}\right)$, but there is no such restriction on the associated negatively curved metrics. We then recover many of the standard results for groups of hyperbolic isometries of $\mathrm{H}^{3}$ in this more general setting under mild and usually necessary restrictions, such things as the limits being discrete, or the deformations are algebraically trivial and so forth.
\end{abstract}

We point out, as a warning, that some authors use the term negatively curved groups to mean hyperbolic groups in the sense of Gromov [G].

The pinching condition we assume may be relaxed if the curvature is nonpositive, bounded below and the associated Hadamard manifold is a visibility manifold [EO]. The version of Jørgensen's inequality $[\mathbf{J}]$ that we produce follows more or less directly as in $[\mathbf{M}]$, where the $n$-dimensional hyperbolic case is considered, from Gromov's generalization of the convergence of iterated commutators, see Buser and Karcher [BK, §2]. The results of this paper can be viewed as further applications of that fundamental result. Other aspects of the theory of isometry groups of negative curvature can be found in the book of Ballman, Gromov and Schroeder [BGS] which we use as a general reference.

In $\S \S 3,4$ we discuss the algebraic limits of negatively curved groups (in the appropriate homeomorphism group) and show that under mild (and necessary) hypotheses they are discrete. We also obtain that a group of isometries of a negatively curved metric is discrete if and only 
if all its two generator subgroups are discrete under either the algebraic assumption that the order of the torsion is bounded, or the geometric assumption that the fixed points of hyperbolics are in general position. It is not difficult to see that some assumption is necessary. Our result includes those negatively curved groups which, as abstract groups, are hyperbolic in the sense of Gromov.

In $\S 5$ we show that continuous deformations of most (for instance those that are virtually torsion free) discrete negatively curved groups are algebraically trivial; that is, the deformation consists entirely of isomorphic groups. (Here we vary the generators continuously in the homeomorphism group, the underlying metrics need not change continuously.) From this it turns out that a continuous deformation of a cocompact torsion free group is topologically trivial; that is, all the groups are topologically conjugate (including the action on the sphere at infinity).

There are three main reasons why our results (except in the $\mathbb{R}$-rank one case where more precise statements can be made) are not as good as the hyperbolic case:

First, in this general setting there is no Selberg Lemma [S] asserting the existence of torsion free subgroups of finite index in finitely generated matrix groups. Indeed there is an example, due to Gromov [G, $\S 4.5 \mathrm{C}]$, of a finitely generated infinite torsion group acting on a space of nonpositive curvature (this space is not a manifold). He remarks, page 79 , that it might be possible to achieve a uniform bound on the order of the torsion and thus produce a geometric Burnside group. It is true however that a purely torsion negatively curved group is finite. This is a simple consequence of [G, Lemma 8.1 A].

Secondly, there is no known compact core theorem, as for instance Scott's Core Theorem for 3-manifolds [Sc]. Thus we cannot assume that a finitely generated group is finitely presented. Indeed, there are finitely generated discrete hyperbolic groups (in dimension $n \geq 4$ ) which are not finitely presented [KP]. We point out that there is a useful version of the core theorem if one assumes pinched curvature and the injectivity radius goes to zero [BGS, Theorem 10.5].

And thirdly, in [GM] we construct an infinite parabolic convergence group which contains free groups of arbitrarily large rank and so is not virtually nilpotent. The Heisenberg group is a purely parabolic group of isometries of complex hyperbolic space which is not virtually abelian. Consequently, parabolicity is not as easy a condition to deal with as it is in the constant curvature case. It should be noted, 
however, that a finitely generated discrete parabolic negatively curved group is virtually nilpotent; see for instance [B] who shows that discreteness implies finite generation in this case.

Finally we remark that it is an interesting feature of our work that the underlying metrics do not play a crucial role in the discreteness of limits. The dependence is more on the topological action of such groups on the sphere at infinity (as convergence groups in the sense of $[\mathbf{G M}]$ ) which enables us to produce the appropriate version of Jørgensen's inequality. The algebraic limits of discrete negatively groups may only be groups of homeomorphisms, but our results imply they are discrete and have reasonable geometric structure.

1. Notation and Definitions. We denote by $\mathbf{B}^{n}$ the closed unit ball of euclidean $n$-space $\mathbb{R}^{n}$. A Hadamard manifold $M$ is a complete simply connected manifold all of whose Riemannian sectional curvatures $K(M) \leq 0$. Every $n$-dimensional Hadamard manifold is diffeomorphic to $\operatorname{int}\left(\mathbf{B}^{n}\right)$ via the usual exponential mapping (CartanHadamard Theorem). The universal covering of any manifold whose sectional curvatures are nonpositive is a Hadamard manifold. Two unit speed geodesics $c_{1}, c_{2}$ in $M$ are asymptotic if there is some constant $b$ such that $d\left(c_{1}(t), c_{2}(t)\right) \leq b$ for all $t \geq 0$. The equivalence classes of asymptotic geodesics are called points at infinity and the collection of all such points is denoted $S_{\infty}$. There is a natural topology on $M \cup S_{\infty}$ which makes it homeomorphic to $\mathbf{B}^{n}$ [EO]. If the sectional curvatures are strictly negative, $K(M) \leq-a^{2}<0$, then $M$ is a visibility manifold in the sense of [EO]. Then for distinct $x$ and $y$ in $S_{\infty}$ there is a unique geodesic $c: \mathbb{R} \rightarrow M$ such that $c(+\infty)=x$ and $c(-\infty)=y$. We say that a Hadamard manifold $M$ has pinched curvature if all of the sectional curvatures of $M$ satisfy

$$
-1 \leq K(M) \leq-a^{2},
$$

where $a \neq 0$. The choice of -1 on the left-hand side of the above inequality is a normalization which can be achieved by scalar multiplication of the Riemannian metric as soon as the sectional curvatures satisfy $-B^{2} \leq K(M) \leq-b^{2}$. It is easy to see that any isometry of a pinched Hadamard manifold $M$ extends to a homeomorphism of $M \cup S_{\infty}$ via its action on geodesics.

It is clear from the above discussion that the study of pinched Hadamard manifolds and their isometries is equivalent to the study of Riemannian metrics $g$ defined on the open unit ball of $\mathbb{R}^{n}$ and for which all the sectional curvatures satisfy $-1 \leq K(g) \leq-a^{2}$. For 
simplicity, this is the framework in which we will work. We say a group $\Gamma$ of homeomorphisms of $\mathbf{B}^{n}$ is a negatively curved group if there is some metric $g$ satisfying the above pinching condition and for which $\Gamma$ acts as a group of isometries.

We say that a negatively curved group $\Gamma$ is discrete if the identity is isolated in $\Gamma$ (the topology here is essentially irrelevant). Discrete negatively curved groups arise naturally in the study of manifolds with pinched curvature. If $(M, g)$ is a Riemannian manifold with pinched sectional curvature, then the fundamental group $\pi_{1}(M)$ is a negatively curved discrete group. Discrete subgroups of $\mathbb{R}$-rank one Lie groups are negatively curved groups. Let $\gamma$ be an isometry of a pinched curvature metric $g$ with associated distance function $d(\cdot, \cdot)$. For $x \in \operatorname{int}\left(\mathbf{B}^{n}\right)$ we define $d_{\gamma}(x)=d(\gamma(x), x)$ and $d_{\gamma}=\inf \left\{d_{\gamma}(x): x \in\right.$ $\left.\operatorname{int}\left(\mathbf{B}^{n}\right)\right\} ; d_{\gamma}$ is called the translation length of $\gamma$. The isometry $\gamma$ is classified by its translation length. We say $\gamma$ is elliptic if $d_{\gamma}=0$ and this infimum is attained, $\gamma$ is hyperbolic if $d_{\gamma}>0$ and this infimum is attained, and $\gamma$ is parabolic if the infimum is not obtained. If $\gamma$ is elliptic, then the fixed point set of $\gamma$ in $\operatorname{int}\left(\mathbf{B}^{n}\right)$ is a nonempty complete totally geodesic subspace of codimension at least 1 (actually 2 if $\gamma$ is orientation preserving). If $\gamma$ is hyperbolic, then $\gamma$ has two fixed points on the sphere at infinity. The unique geodesic connecting these two points is completely invariant and $\gamma$ translates along the geodesic by the distance $d_{\gamma}$. If $\gamma$ is parabolic, then $\gamma$ has a single fixed point on the sphere at infinity [BGS]. This characterization implies the notion of hyperbolicity, parabolicity and ellipticity are well defined for negatively curved groups. That is, they are independent of the underlying metric (of which there may be many).

In [MS] we prove that a discrete negatively curved group is a convergence group in the sense of [GM]. These are groups of homeomorphisms with the compactness properties of quasiconformal (and hence conformal) mappings. More precisely a group $\Gamma$ of homeomorphisms of $\mathbf{B}^{n}$ is called a convergence group if every infinite family of elements of $\Gamma$ contains a sequence $\left\{\gamma_{i}\right\}_{i \geq 1}$ for which there are $x$ and $y$ in $\mathbf{S}^{n-1}$ such that

$$
\gamma_{i} \rightarrow x \text { locally uniformly in } \mathbf{B}^{n}-\{y\}
$$

and

$$
\gamma_{i}^{-1} \rightarrow y \text { locally uniformally in } \mathbf{B}^{n}-\{x\},
$$

the possibility $x=y$ may occur. This definition is actually that of a discrete convergence group in our reference [GM], but we adopt this 
terminology for simplicity. Note too that in accordance with the usual terminology for isometries of negatively curved spaces we use the term hyperbolic for loxodromic elements.

The limit set, $L(\Gamma)$, of a convergence group $\Gamma$ is the set of accumulation points of $\Gamma(0)=\{\gamma(0): \gamma \in \Gamma\}$. We say that $\Gamma$ is elementary if the limit set contains fewer than three points. Otherwise the limit set is a perfect set, and the fixed points of hyperbolic elements are pairwise dense in $L(\Gamma) \times L(\Gamma)$. In [GM] we classify the elementary convergence groups. The elements in a convergence group are also classified according to their fixed point data and their order. In the case of a discrete group of isometries of a pinched metric, the above implies that the two classifications correspond.

Let $g$ be a negatively curved Riemannian metric on $\operatorname{int}\left(\mathbf{B}^{n}\right), d(\cdot, \cdot)$ the associated distance function, $\gamma$ an isometry and $x \in \operatorname{int}\left(\mathbf{B}^{n}\right)$. Then the rotation of $\gamma$ at $x$ is the angle

$$
r_{\gamma}(x)=\max \left\{\angle\left(w, P_{\gamma}\left(\gamma_{* x}(w)\right)\right): w \in T_{x} \mathbf{B}^{n} \text { and }|w|=1\right\} .
$$

Here $\gamma_{* x}: T_{x} \mathbf{B}^{n} \rightarrow T_{\gamma(x)} \mathbf{B}^{n}$ is the differential at $x$ and $P_{\gamma}: T_{\gamma(x)} \mathbf{B}^{n} \rightarrow$ $T_{x} \mathbf{B}^{n}$ is parallel transportation along the geodesic from $x$ to $\gamma(x)$. We then define the norm of $\gamma$ at $x$ as

$$
n_{\gamma}(x)=\max \left\{r_{\gamma}(x), 8 d_{\gamma}(x)\right\} \text {. }
$$

One of the most important results we use in the following consequence of the generalization (due to Gromov) of the convergence of iterated commutators in Lie groups, see Corollary 2.4.4 of [BK]. This result can be found in [BGS, Corollary p. 106].

1.1. TheOREM. Let $g$ be a pinched Riemannian metric, $x \in$ $\operatorname{int}\left(\mathbf{B}^{n}\right)$ and $N$ a discrete group of isometries generated by elements $\alpha$ with $n_{\alpha}(x) \leq 0.49$. Then $N$ is nilpotent.

It is interesting to note that the constant 0.49 is independent of dimension. In the three-dimensional hyperbolic case, a related result can be proved purely in terms of the translation length [GM2, §5].

It is easy to prove that a nonelementary convergence group contains a subgroup isomorphic to the free group on two generators and is therefore not nilpotent. Thus in Theorem 1.1 we could have arranged the (weaker) conclusion that $N$ is elementary and so in particular the limit set consists of at most two points.

2. Jørgensen's inequality for negative curvature. In this section we prove a generalization of Jørgensen's inequality for Kleinian groups 
[J]. This result can be seen as a generalization of the classical Margulis lemma, or the existence of Zassenhaus neighbourhoods. First we need a couple of lemmas. We assume that $\gamma$ is an isometry of a negatively curved metric. A line is a complete geodesic. We fix $x \in \operatorname{int}\left(\mathbf{B}^{n}\right)$.

2.1. LEMMA. If $\gamma$ interchanges the endpoints of a line, then $\gamma$ is elliptic and $n_{\gamma}(x) \geq \pi / 2$.

Proof. If $\gamma$ interchanges the endpoints of a line, then $\gamma^{2}$ has two additional fixed points and hence is elliptic. Thus $\gamma$ too is elliptic. Next, $\gamma$ has a fixed point on this line and so the set $V$ of perpendicular geodesics to this line and passing through the fixed point is invariant and separates $\mathbf{B}^{n}$ into two components (if there were more components we would find conjugate points). One of these components $V^{+}$contains $x$, the other $V^{-}$contains $\gamma(x)$. Let $C$ be the geodesic joining $x$ to $\gamma(x)$ and $y$ the point of intersection of $C$ and $V$. Let $w$ be the unit tangent vector at $x$ whose parallel transport along $C$ to $y$ is perpendicular to $V$ and points into $V^{+}$. Then $\gamma *_{x}(w)$ points in the direction of $V^{-}$as does its parallel transport to $y$ (note that $\gamma^{2}(x) \in V^{+}$). Thus the angle between the parallel transports of $w$ and $\gamma *_{x}(w)$ to $y$ is at least $\pi / 2$. This establishes the lemma.

The following two lemmas are proved in a manner similar to Lemmas 4.1 and 4.2 of [M]. We illustrate the proof of the second lemma.

2.2. LemMA. Suppose $\alpha$ and $\beta$ are two isometries generating a discrete negatively curved group. If $\alpha$ is parabolic or hyperbolic and if the group $\left\langle\alpha, \beta \alpha \beta^{-1}\right\rangle$ is elementary, then the group $\langle\alpha, \beta\rangle$ is also elementary.

2.3. LEMMA. Let $\alpha$ and $\beta$ be two isometries generating a discrete negatively curved group. Suppose that $\alpha$ is elliptic and that $p$ is the dimension of the fixed point set of $\alpha$. If the group

$$
\Gamma=\left\langle\beta^{i} \alpha \beta^{-i}: i=0,1,2, \ldots, p+1\right\rangle
$$

is elementary, then either $\langle\alpha, \beta\rangle$ is elementary or $n_{\alpha}(x) \geq \pi / 2$.

Proof. Let $V=\mathrm{fix}(\alpha)$. As we have noted earlier $V$ is a nonempty complete totally geodesic subspace. Given a collection of points $x_{0}$, $x_{1}, \ldots, x_{m}$ we denote by $\operatorname{sp}\left(x_{0}, x_{1}, \ldots, x_{m}\right)$ the smallest totally 
geodesic complete subset containing these points. The curvature assumption easily implies that $\operatorname{sp}\left(x_{0}, \ldots, x_{m}\right)$ is diffeomorphic (for instance via the exponential mapping) to the interior of a ball of some dimension at most $n$ and that the closure of this set is topologically a ball. As with the hyperbolic case we need to consider three cases.

(1) Suppose $\Gamma$ is finite. Then there is a nonempty complete totally geodesic subspace $F$ pointwise fixed by every element of $\Gamma$. Let $w \in$ $F$. Then $\beta^{i} \alpha \beta^{-i}(w)=w$ for all $i=0,1,2, \ldots, p+1$ and so $x_{i}=$ $\beta^{-i}(w) \in V$ for all $i=1, \ldots, p+1$. Let $j$ be the smallest integer such that $\operatorname{sp}\left(x_{0}, x_{1}, \ldots, x_{j}\right)=\operatorname{sp}\left(x_{0}, x_{1}, \ldots, x_{j}, x_{j+1}\right)$. Such a $j$ exists by general position as the $\left\{x_{i}\right\}$ are a collection of $p+2$ points lying in the $p$-dimension subspace $V$. As $\beta$ is an isometry we have

$$
\begin{aligned}
\beta\left(\operatorname{sp}\left(x_{0}, x_{1}, \ldots, x_{j}\right)\right) & =\operatorname{sp}\left(\beta\left(x_{0}\right), \beta\left(x_{1}\right), \ldots, \beta\left(x_{j}\right)\right) \\
& =\operatorname{sp}\left(x_{1}, x_{2}, \ldots, x_{j+1}\right) .
\end{aligned}
$$

But $\operatorname{sp}\left(x_{1}, x_{2}, \ldots, x_{j+1}\right)$ is a subspace of $\operatorname{sp}\left(x_{0}, x_{1}, x_{2}, \ldots, x_{j+1}\right)$ and a dimension count implies

$$
\beta\left(\operatorname{sp}\left(x_{0}, x_{1}, \ldots, x_{j}\right)\right)=\operatorname{sp}\left(x_{0}, x_{1}, \ldots, x_{j}\right) .
$$

Since the closure of the set is topologically a ball, $\beta$ has a fixed point in $\operatorname{sp}\left(x_{0}, x_{1}, \ldots, x_{j}\right)$, a subset of $V$, and therefore $\langle\alpha, \beta\rangle$ fixes a point and must be an elementary group [GM].

(2) Suppose $L(\Gamma)=\left\{x_{0}\right\}$. Since the limit set is $\Gamma$ invariant we have $\alpha\left(x_{0}\right)=x_{0}$ and for all $i=1,2, \ldots, p+1, \beta^{i} \alpha \beta^{-i}\left(x_{0}\right)=x_{0}$. The above argument now applies.

(3) Suppose $L(\Gamma)=\left\{x_{0}, y_{0}\right\}$. Again, the limit set is $\Gamma$ invariant and so every element either fixes or interchanges the set $\left\{x_{0}, y_{0}\right\}$. If every element of $\Gamma$ fixes this set, the argument of part one applies to give the result. Otherwise there is an element of $\Gamma$ which interchanges these points and therefore the line between them. Consequently one of the generators must have this property. Since they are all conjugates of $\alpha, \alpha$ must have this property. Then Lemma 2.1 implies $n_{\alpha}(x) \geq \pi / 2$.

The following is the necessary generalization of Jørgensen's inequality.

2.4. TheOREM. Suppose that $\alpha$ and $\beta$ generate a discrete nonelementary negatively curved group. If $\alpha$ is hyperbolic or parabolic, then for each $x \in \operatorname{int}\left(\mathbf{B}^{n}\right)$

$$
\begin{aligned}
& \max \left\{n_{\alpha}(x), n_{[\alpha, \beta]}(x)\right\} \geq 0.49 \text { and } \\
& \max \left\{n_{\alpha}(x), n_{\beta \alpha \beta-1}(x)\right\} \geq 0.49 .
\end{aligned}
$$


If $\alpha$ is elliptic and $p=\operatorname{dim}(\operatorname{fix}(\alpha))$, then

$$
\begin{aligned}
& \max \left\{n_{\alpha}(x), n_{\left[\alpha, \beta^{l}\right]}(x): i=1,2, \ldots, p+1\right\} \geq 0.49 \text { and } \\
& \max \left\{n_{\beta^{l} \alpha \beta^{-l}}(x): i=0,1,2, \ldots, p+1\right\} \geq 0.49
\end{aligned}
$$

Proof. Suppose that the inequality does not hold. In the parabolic or hyperbolic case we find from Theorem 1.1 that the group $\left\langle\alpha, \beta \alpha \beta^{-1}\right\rangle$ is nilpotent and hence elementary. The result in this case follows by Lemma 2.2. In the elliptic case, we again obtain from Theorem 1.1 that the group $\left\langle\beta^{i} \alpha \beta^{-i}: i=0,1, \ldots, p+1\right\rangle$ is elementary. The result then follows by Lemma 2.3 .

It is the following principle, first espoused by Jørgensen, that makes Theorem 2.4 such a valuable tool.

If two isometries $\alpha, \beta$ of a negatively pinched metric generate a nonelementary discrete group, then given $\alpha, \beta$ cannot be too close to the identity.

Jørgensen's inequality is conjugacy invariant, while the above are not (because of the dependence on $x$ ). However a simple corollary is

2.5. Corollary. Suppose that $\alpha$ and $\beta$ generate a discrete nonelementary negatively curved group. If $\alpha$ is hyperbolic or parabolic,

$$
\inf \left\{\max \left\{n_{\alpha}(x), n_{[\alpha, \beta]}(x)\right\}: x \in \operatorname{int}\left(\mathbf{B}^{n}\right)\right\} \geq 0.49 \text {. }
$$

There is of course a corresponding result if $\alpha$ is elliptic.

3. A limit theorem. We recall here that even in the hyperbolic case, for dimensions greater than three, the limit of finitely generated discrete nonelementary groups may not be discrete (even if the limit is assumed to be nonelementary) $[\mathbf{M}, \S 5]$. The problem arises as there may be elliptics of high order converging to an irrational rotation about a codimension two or larger subspace stabilized by a nonelementary discrete subgroup. Thus in $[\mathbf{M}]$ we had to introduce the notion of uniformly bounded torsion. We must of course make a similar assumption in this more general case. To begin with we need the following lemma, essentially due to Newman [N], which we leave the reader to verify. It can be proved using Newman's result and a compactness argument for near the fixed point set the rotation angle is proportional to the reciprocal of the order.

3.1. Lemma. There is a positive constant $\delta=\delta(m, n)$ such that if $x \in \operatorname{int}\left(\mathbf{B}^{n}\right)$ and $\alpha$ is a periodic isometry of period less than or equal 
to $m$ (which is not the identity), then

$$
n_{\alpha}(x) \geq \delta(m)
$$

3.2. Definition. Let $\left\{\Gamma_{i}\right\}_{i \geq 0}$ be a family of groups. We say that $\left\{\Gamma_{i}\right\}$ has uniformly bounded torsion if there is an integer $N$ such that for all $i$ and $\gamma \in \Gamma_{i}$, either $\operatorname{ord}(\gamma)=\infty$ or $\operatorname{ord}(\gamma) \leq N$. Here $\operatorname{ord}(\gamma)$ is the minimal $m \neq 0$ such that $\gamma^{m}=$ identity.

If $\Gamma$ is group of homeomorphisms of $\mathbf{B}^{n}$ (possibly not discrete) we say that $\Gamma$ is nonelementary if there are two elements $\alpha$ and $\beta$ for which

(a) $\alpha^{2}$ and $\beta^{2}$ have disjoint fixed point sets (if one has finite order) or

(b) $\alpha$ and $\beta$ are of infinite order and $\alpha^{2}$ and $\beta^{2}$ have different fixed point sets.

By different we mean that they do not coincide exactly. Otherwise $\Gamma$ is elementary. This apparently weaker definition of nonelementary is equivalent to the usual definition for finitely generated Kleinian groups. We make this definition so as to include infinite torsion groups without a common fixed point amongst the nonelementary groups. We point out that if in addition $\Gamma$ is a convergence group, then (a) and (b) follow from the definition of nonelementary given earlier. As (a) and (b) are essentially all that is needed in the limit groups for our proofs we shall use this definition henceforth.

Here is the first convergence theorem. It is analogous to [ $J$, Proposition 1].

3.3. THEOREM. Let $\Gamma$ be a nonelementary group of homeomorphisms of $\mathbf{B}^{n}$. For each $m \geq 0$ let $\psi_{m}$ be a mapping $\psi_{m}: \Gamma \rightarrow$ Isom $\left(\mathbf{B}^{n}, g_{m}\right)$ into the isometry group of a Riemannian metric $g_{m}$ whose sectional curvatures satisfy $-1 \leq K\left(g_{m}\right) \leq-a_{m}^{2}<0$ and which has discrete image. Suppose that $\left\{\psi_{m}(\Gamma)\right\}_{m \geq 0}$ has uniformly bounded torsion and that for each $\gamma \in \Gamma$

$$
\psi_{m}(\gamma) \rightarrow \gamma
$$

uniformly in $\mathbf{B}^{n}$ as $m \rightarrow \infty$. Then $\Gamma$ is discrete.

Proof. Suppose that $\Gamma$ is not discrete. Then there is a sequence of elements $\left\{\alpha_{i}\right\}_{i \geq 0}$ converging (uniformly) to the identity in $\Gamma$. From our hypothesis there are two elements $\beta_{1}$ and $\beta_{2}$ satisfying (3.2) (a) or (b). As the fixed point sets are closed, they are uniformly separated. 
Let $x \in \operatorname{int}\left(\mathbf{B}^{n}\right)$. It follows by continuity that for $j=1,2$ and sufficiently large $i$ and $m$ that

$$
n_{\psi_{m}\left(\alpha_{t}\right)}(x)+n_{\left[\psi_{m}\left(\alpha_{\imath}\right), \psi_{m}\left(\beta_{\jmath}\right)\right]}(x) \leq \min \{\delta(N), 0.49\} .
$$

Where $N$ is the bound on the maximal order of the cyclic subgroups and $\delta(N)$ is the number from Lemma 3.1. Thus $\psi_{m}\left(\alpha_{i}\right)$ is not elliptic for $m$ and $i$ sufficiently large and so from Theorem 2.4 the groups $\left\langle\psi_{m}\left(\alpha_{i}\right), \psi_{m}\left(\beta_{j}\right) \psi_{m}\left(\alpha_{i}\right) \psi_{m}\left(\beta_{j}\right)^{-1}\right\rangle, j=1,2$, are elementary and have nonempty limit set. If the limit set consists of one point, then these two elements have a common fixed point which is stabilized by $\psi_{m}\left(\beta_{j}\right), j=1,2$. If the limit set contains two points, then $\psi_{m}\left(\beta_{j}\right)$ fixes or interchanges this set, $j=1,2$ and the squares have a common fixed point. Now letting $m \rightarrow \infty$ we obtain the desired contradiction.

REMARK. Of course in the hyperbolic case the assumption of uniform convergence implies that the limits are again hyperbolic isometries. Notice too that in the above situation we make no assumption about the convergence of the metrics $\left\{g_{m}\right\}_{m \geq 0}$, and that in the limit $\Gamma$ may not even be a group of diffeomorphisms. Presumably however there is some underlying metric structure in the limit.

3.4. CoROllary. Let $\Gamma$ be a nonelementary group of isometries of a Riemannian metric all of whose sectional curvatures satisfy $-A^{2} \leq$ $K(g) \leq-a^{2}$, which has bounded torsion. Then $\Gamma$ is discrete if and only if every two generator subgroup is discrete.

Proof. By multiplying the metric by a suitable constant we may assume the curvature satisfies $-1 \leq K(g) \leq-c^{2}<0$. Suppose that $\Gamma$ is not discrete and let $\gamma_{i} \rightarrow$ identity in $\Gamma$. Since $\Gamma$ has bounded torsion we may assume $\gamma_{i}$ is not elliptic. By hypothesis there are two elements $\beta_{j}, j=1,2$, which are parabolic or hyperbolic and whose fixed point sets do not coincide exactly, or the fixed point sets are disjoint. Also by hypothesis the sequence of groups $\left\langle\gamma_{i}, \beta_{j}\right\rangle, j=$ 1,2 , is discrete. Finally an argument similar to Theorem 3.3 implies this sequence of groups is elementary for sufficiently large $i$. We therefore reach a contradiction as above and this establishes the result.

3.5. Corollary. Let $\Gamma$ be a group of isometries of a Riemannian metric all of whose sectional curvatures satisfy $-A^{2} \leq K(g) \leq-a^{2}$. Suppose that the fixed points of hyperbolic elements of $\Gamma$ are in general position (that is, the fixed points of hyperbolic elements do not lie in a 
totally geodesic codimension 1 set). Then $\Gamma$ is discrete if and only if every two generator subgroup is discrete.

Proof. Let $\beta_{j}, j=1,2, \ldots, k$ be hyperbolic elements whose fixed point sets are in general position. Let $\left\{\gamma_{i}\right\}$ be a sequence as in Corollary 3.4. If this sequence (or some subsequence) is not eventually elliptic we can argue as above and conclude the desired result. Thus we assume $\gamma_{i}$ are all elliptic. Then for all $j$ and for all $i$ sufficiently large we have (as above) $\left\langle\gamma_{i}, \beta_{j}\right\rangle$ is a discrete elementary group and so for all $j, \operatorname{fix}\left(\beta_{j}\right)$ lies in $\operatorname{fix}\left(\gamma_{i}\right)$ as $\beta_{j}$ is hyperbolic. This is impossible as $\operatorname{fx}\left(\gamma_{i}\right)$ is totally geodesic and codimension at least 1 .

In the above two results it is clear that some additional hypothesis, such as bounded torsion or the constraint on the fixed points of hyperbolic elements, is necessary (even in the hyperbolic case). We might for instance encounter a negatively curved group isomorphic to $\Gamma \times \mathbf{S}$, with the circle action stabilizing a lower dimensional hyperbolic space on which $\Gamma$ acts discretely. If $\mathbf{Q}$ is that subgroup of $\mathbf{S}$ containing all the elements of finite order, then $\Gamma \times \mathbf{Q}$ has the property that all its two generator subgroups are discrete. Of course $\Gamma \times \mathbf{Q}$ is not discrete. However, it is quite possible that the additional hypotheses are redundant in the case that the group is finitely generated (this of course implies bounded torsion by Selberg's lemma in the symmetric cases). Thus we ask the question: is it true that finitely generated groups of isometries of a metric of pinched negative curvature have bounded torsion? G. Mess showed me an argument which implies this is the case if the abstract group is hyperbolic in the sense of Gromov [G].

\section{Algebraic convergence.}

4.1. Definition. Let $\left\{\Gamma_{i}\right\}_{i \geq 0}$ be a sequence of negatively curved groups each with the same finite number of generators $\left\{\gamma_{i, 1}, \gamma_{i, 2}, \ldots\right.$, $\left.\gamma_{i, m}\right\}$. If for each $j=1,2, \ldots, m$, there is a self homeomorphism $\gamma_{j}$ of $\mathbf{B}^{n}$ such that

$$
\gamma_{i, j} \rightarrow \gamma_{j} \text { as } i \rightarrow \infty,
$$

then we say that the groups $\Gamma_{i}$ converge algebraically to the group $\Gamma=\left\langle\gamma_{1}, \gamma_{2}, \ldots, \gamma_{m}\right\rangle$.

REMARK. Again we note that there is no assumption on the convergence of the underlying Riemannian metrics of negative curvature, and moreover no assumption that their curvature is uniformly bounded above by a negative constant. Thus the limit could possibly be a group 
of isometries of a flat metric (and therefore a negatively curved group). It is a consequence of our results that this is not the case.

4.2. THEOREM. Let $\Gamma$ be a group of homeomorphisms of $\mathbf{B}^{n}$ which is the algebraic limit of a sequence $\left\{\Gamma_{i}\right\}_{i \geq 0}$ of nonelementary negatively curved groups with uniformly bounded torsion. Then $\Gamma$ is infinite and discrete. Moreover, if $\Gamma$ is a negatively curved group, then $\Gamma$ is nonelementary.

In the three-dimensional hyperbolic case the assumption of bounded torsion is unnecessary and also that $\Gamma$ is nonelementary is a consequence of algebraic convergence [JK]. For the higher-dimensional hyperbolic case, the assumption of bounded torsion (or a related assumption) is necessary and also implies that $\Gamma$ is nonelementary. Notice in Theorem 4.2 we have skirted the delicate issue of when the limit of negatively curved groups is again negatively curved. We hope to return to this at a later date.

Proof. Let

$$
\Gamma_{i}=\left\langle\gamma_{i, 1}, \gamma_{i, 2}, \ldots, y_{i, m}\right\rangle \text { with } \gamma_{i, j} \rightarrow \gamma_{i}
$$

be a sequence as in Theorem 4.2 with $\Gamma=\left\langle\gamma_{1}, \gamma_{2}, \ldots, \gamma_{m}\right\rangle$. We proceed by a series of lemmas.

\subsection{LEMMA. $\Gamma$ is not finite.}

Proof. Suppose $\Gamma$ is finite. Then there is a $0 \leq k<\infty$ such that every element of $\Gamma$ can be expressed as a word in the generators $\left\{\gamma_{i}\right\}$ of word length at most $k$. Since $\Gamma_{i}$ is nonelementary, there are words of all lengths. For each $i$ choose a word $w_{i}$ of length $k+1$ in $\Gamma_{i}$ (in terms of the generators given). Since there are finitely many such words, passing to subsequence we may assume that the sequence of words converges to a word $w$ in $\Gamma$. This limit word can be expressed in a word $w^{\prime}$ of length $k$, and then let $w_{i}^{\prime}$ be the corresponding word in $\Gamma_{i}$. Then the sequence of words $v_{i}=w_{i} w_{i}^{\prime-1}$ is nontrivial, has word length at most $2 k+1$ and converges to the identity. The assumption of bounded torsion implies that $v_{i}$ is not elliptic for: $i$ sufficiently large by Lemma 3.1. Thus $v_{i}$ is parabolic or hyperbolic. Then there is a generator $\gamma_{i, j}$ which does not stabilize the set $\operatorname{fix}\left(v_{i}\right)$. Hence the group $\left\langle v_{i}, \gamma_{i, k}\right\rangle$ is nonelementary. But as $v_{i} \rightarrow$ identity and $\gamma_{i j} \rightarrow \gamma_{j}$, we easily obtain a contradiction to Corollary 2.5. 
Hence $\Gamma$ is finitely generated and infinite. In the classical case (or whenever $\Gamma$ is isomorphic to a subgroup of $\operatorname{GL}(m, \mathbb{C})$ for some $m)$ we could now use Selberg's Lemma [S] to assert the existence of elements of infinite order. This would simplify the following arguments.

\subsection{LEMMA. $\Gamma$ is discrete.}

Proof. If not choose $\alpha \in \Gamma$, so close to the identity (but $\alpha \neq$ identity) so that if $\alpha_{i}$ is any approximant to $\alpha$ in $\Gamma_{i}$, then $\alpha_{i}$ is parabolic or hyperbolic and also that for some $x \in \operatorname{int}\left(\mathbf{B}^{n}\right)$

$$
n_{\alpha_{t}}(x)+n_{\gamma_{\imath, j} \alpha_{\imath} \gamma_{t, j}^{-1}}(x)<0.49, \quad j=1,2, \ldots, m .
$$

We can of course do this by appealing to continuity and Lemma 3.1. Then the group $\left\langle\alpha_{i}, \gamma_{i, j} \alpha_{i} \gamma_{i, j}^{-1}\right\rangle$ is elementary by Corollary 2.5. Thus each generator stabilizes $\operatorname{fix}\left(\alpha_{i}\right)$ and the group they generate is elementary. This is contrary to our hypothesis.

We suppose henceforth that $\Gamma$ is a negatively curved group.

\subsection{LEMMA. $\Gamma$ is nonelementary.}

Proof. As $\Gamma$ is infinite and negatively curved, there is a hyperbolic or parabolic $\alpha$ in $\Gamma$ ([G, Lemma 8.1 A] implies the group is not purely torsion). Since $\Gamma_{i}$ is nonelementary, some generator $\gamma_{i, k}$ does not setwise fix the fixed points of $\alpha_{i}$. Passing to a subsequence we may assume that $k=1$ and $\gamma_{i, 1}=\gamma_{i}$. If $\gamma$ and $\alpha$ do not have a common fixed point, then we are done and so we suppose otherwise. Now suppose $\langle\alpha, \gamma\rangle$ is discrete and elementary. If $\alpha$ is hyperbolic, then by [GM] the group $\langle\alpha, \gamma\rangle$ is virtually cyclic. This easily leads to a contradiction. We are left to consider the case that every element of $\Gamma$ of infinite order is parabolic with the same fixed point [GM]. That is, $\Gamma$ is a finitely generated negatively curved parabolic group and so is virtually nilpotent [B]. Then there are integers $p$ and $q$ such that $a=\alpha^{p}$ and $b=\gamma \alpha^{q} \gamma^{-1}$ generate a nilpotent group. Let $x \in \operatorname{int}\left(\mathbf{B}^{n}\right)$. There is a $d$ such that any $d$-fold commutator [, $]_{d}$ of $a$ and $b$ is trivial. Thus, summing over all the finitely many $d$-fold commutators involving only $a$ and $b$

$$
\sum_{[,]_{d}} n_{[,]_{d}}(x)=0 \text {. }
$$

Then by continuity and Theorem 1.1 , for sufficiently large $i$, the group generated by the $d$-fold commutators of the approximants $a_{i}$ and $b_{i}$ 
will be nilpotent. Thus there is a $d^{\prime}$ so that all $d^{\prime}$-fold commutators involving (only) the approximants $a_{i}$ and $b_{i}$ are trivial. Then the commutator identity $[a, b c]=[a, b][b,[a, c]][a, c]$ implies that all $d^{\prime}$-fold commutators are trivial as they can be written as (much longer) products of $d^{\prime}$-fold commutators in $a_{i}$ and $b_{i}$, see for instance [BK, p. 28]. Thus $\left\langle a_{i}, b_{i}\right\rangle$ is eventually nilpotent and hence elementary. But this is easily seen not to be the case. The contradiction establishes the lemma.

Actually, the above argument only needs that $\Gamma$ is a convergence group which is not purely torsion and whose discrete parabolic subgroups are virtually nilpotent. If this is actually the case, we can further restrict the possibilities for $\Gamma$ with the following

4.6. LEMMA. If $\Gamma$ is infinite and purely torsion convergence group, then $\Gamma$ is discrete and has bounded torsion.

Proof. $\Gamma$ is discrete. If there is no bound on the order of torsion elements, then the approximants to all elements of sufficiently high order will have to be parabolic or hyperbolic. Powers of these elements will again be parabolic or hyperbolic and close to the identity. The argument of Lemma 4.4 again produces a contradiction.

4.7. TheOREM. Let $\Gamma$ be a finitely generated abstract group with uniformly bounded torsion. For each $m \geq 0$ let

$$
\psi_{m}: \Gamma \rightarrow \operatorname{Isom}\left(\mathbf{B}^{n}, g_{m}\right), \quad-1 \leq K\left(g_{m}\right) \leq-a_{m}^{2}<0,
$$

be an isomorphism such that the images $\psi_{m}(\Gamma)=\Gamma_{m}$ are discrete and nonelementary and converge algebraically to $\Gamma_{\infty}$. Then $\Gamma_{\infty}$ is discrete and the correspondence of generators induces an isomorphism $\psi_{\infty}: \Gamma_{\infty} \rightarrow \Gamma$.

Proof. As $\psi_{m}$ is an isomorphism, the sequence $\left\{\Gamma_{m}\right\}_{m \geq 0}$ has uniformly bounded torsion. Thus from Theorem $4.2 \Gamma_{\infty}$ is discrete. It is clear that the correspondence of generators $\psi_{\infty}: \Gamma_{\infty} \rightarrow \Gamma \cong \Gamma_{m}$ induces a homomorphism onto. Suppose $\alpha \in \Gamma_{\infty}-\{$ Identity $\}$ and $\psi_{\infty}(\alpha)=$ Identity. Uniformly bounded torsion implies that $\psi_{m(}(\alpha)$ is parabolic or hyperbolic for all $m$ sufficiently large as $\psi_{m}(\gamma) \rightarrow$ Identity by Lemma 3.1. Now apply the argument of Lemma 4.4.

Indeed the above argument easily implies (see [M, Theorem 6.1]). 
4.8. Theorem. Let $\left\{\Gamma_{i}\right\}_{i \geq 0}$ be a sequence of discrete nonelementary negatively curved groups with uniformly bounded torsion converging algebraically to a finitely presented group of homeomorphisms $\Gamma$. Then $\Gamma$ is discrete and the correspondence of generators $\Gamma \rightarrow \Gamma_{l}$ induces a homomorphism for all $i$ sufficiently large.

If each $\Gamma_{i}$ has a finite presentation, each relation of which has uniformly bounded wordlength, then the correspondence eventually induces an isomorphism.

From [BGS, Theorem 10.2] if $\Gamma$ is a negatively curved torsion free group with the injectivity radius of the quotient manifold $\mathbf{B}^{n} / \Gamma$ going to zero at infinity (for instance cofinite volume), then this quotient is diffeomorphic to the interior of a compact manifold (with boundary) and so the fundamental group $\Gamma$ is finitely presented. The conclusion of Theorem 4.7 would then hold and all groups sufficiently close to $\Gamma$, in the topology of algebraic convergence, would be factors of $\Gamma$.

The symmetric Riemannian $\mathbb{R}$-rank one spaces of negative curvature are real hyperbolic space $\mathrm{SO}(1, n) / \mathrm{SO}(n)$, complex hyperbolic space $\mathrm{SU}(1, n) / \mathrm{U}(n)$, quaternionic hyperbolic space $\operatorname{Sp}(1, n) / \operatorname{Sp}(n)$ and hyperbolic space over the Cayley numbers $F_{4} / \operatorname{Spin} 9$. The isometry groups are the Lie groups $\mathrm{SO}(1, n), \mathrm{SU}(1, n), \mathrm{Sp}(1, n)$ and $F_{4}$ respectively. The metric is the canonical left invariant metric with $-1 \leq K \leq-\frac{1}{4}$, see [Mo] and $[\mathbf{H}]$.

Let $\Gamma$ be a discrete subgroup of one of the isometry groups above and let $\mathbf{H}(\Gamma)$ be the geodesically convex hull of the limit set $L(\Gamma)$ of $\Gamma$. We say that $\Gamma$ is of compact type if $\mathbf{H}(\Gamma) / \Gamma$ is compact. Clearly compact type groups are finitely generated and finitely presented. Therefore and application of Theorem 4.8 yields

4.9. Corollary. Suppose $\Gamma$ is a compact type discrete subgroup of the isometry group of an $\mathbb{R}$-rank one space of negative curvature. Then there is a neighbourhood of the generators of $\Gamma$ in $\mathfrak{G} \times \mathfrak{G} \times \cdots \times \mathfrak{G}$ such that every discrete group whose generators lie in this neighbourhood is a factor of $\Gamma$.

5. Continuous deformations. In this section we shall show that a continuous deformation of a negatively curved group, through discrete negatively curved groups, is algebraically trivial, Theorem 5.3. That is, all the groups have the same isomorphism type. It then follows that in the torsion free cocompact case that such a deformation is topologically trivial as well. That is, all the associated quotients 
are homeomorphic and the deformation is induced by a topological conjugacy on the whole (closed) ball.

5.1. Definition. We say a group $\Gamma$ is virtually torsion free, if $\Gamma$ has a torsion free subgroup of finite index. Let $D(k, n)$ denote the space of all virtually torsion free $k$ generator discrete nonelementary negatively curved groups. We give $D(k, n)$ the topology of algebraic convergence. In what follows we fix $x \in \operatorname{int}\left(\mathbf{B}^{n}\right)$ and define the norm of $\alpha$ as $n(\alpha)=n_{\alpha}(x)$.

The proof of the following lemma is more or less implicit in what we have done in $\S 4$, see for instance [M, Lemma 6.2]

5.1. Lemma. Let $\Gamma \in D(k, n)$. Then there is a neighbourhood $N_{\varepsilon}(\Gamma)$ of $\Gamma$ such that if $\alpha \in N_{\varepsilon}(\Gamma)$ and $n(\alpha)<\varepsilon$, then $\alpha=$ Identity.

The following is a generalization of [ $\mathbf{J}$, Theorem 3] and [M, Theorem 6.3]. The proof given here follows the latter reference.

5.2. TheOREM. Let $E$ be a connected compact subset of $D(k, n)$. Then $E$ consists entirely of isomorphic groups.

Proof. Let $E_{m}$ denote that subset of $E$ for which the maximal order of a finite cyclic subgroup is exactly $m$. Then $\left\{E_{m}\right\}_{m \geq 0}$ is a disjoint collection, $E_{m}$ is compact by Theorem 4.2 and as each element of $D(k, n)$ is virtually torsion free $E=\bigcup_{m \geq 0} E_{m}$. Let $\Gamma \in E_{m}$ and $R$ a relation in $\Gamma$, and $F_{m}$ that subset of $E_{m}$ with the relation $R$. Lemma 5.1 implies that $F_{m}$ is relatively open; clearly it is also closed and so is a union of components of $E_{m}$. Thus each component of $E_{m}$ consists entirely of isomorphic groups. As $E$ is compact, Sierpinski's Theorem [K, §47III, Theorem 6] (which states that a compact connected set cannot be the countable union of closed disjoint subsets) implies that $E=E_{m}$ for some $m$ and we are done.

It is interesting to note that each component of $E_{0}$ (the torsion free groups) consists entirely of isomorphic groups. Notice too that in the proof we did not use the full hypothesis that every group is virtually torsion free. Only that each group has bounded torsion. Furthermore, although Sierpinski's Theorem is not true for arbitrary closed sets, it is true for the real line. We can then obtain the following slightly different result. Here, by a continuous deformation, we mean that a fixed finite set of generators is being continuously deformed in $\operatorname{Hom}\left(\mathbf{B}^{n}\right)$. The underlying metrics (of negative curvature) need not change continuously. 
5.3. THEOREM. Let $\Gamma$ be a finitely generated nonelementary negatively curved group with bounded torsion. Let $\left\{\Gamma_{t}: t \in \mathbb{R}\right\}$ be a continuous deformation of $\Gamma=\Gamma_{0}$ through discrete nonelementary negatively curved groups. Then $\left\{\Gamma_{t}: t \in \mathbb{R}\right\}$, and its closure in the topology of algebraic convergence in $\operatorname{Hom}\left(\mathbf{B}^{n}\right)$, consists entirely of isomorphic groups.

Proof. Since the elliptics have integer-valued order, one cannot continuously change this without the order becoming infinite. Since each group in the deformation is assumed discrete, if the order of an elliptic is not preserved by the deformation it must be perturbed to a parabolic or hyperbolic element. But then some power of these elements will be arbitrarily close to the identity (by continuity) and still parabolic or hyperbolic. Pairing this power with its conjugate by each generator will imply by Corollary 2.5 , as we have seen before in Lemma 4.4, that the groups generators stabilize a set containing at most two points and therefore the group they generate is elementary, contrary to our hypothesis. Thus the deformation preserves the orders of elliptics. As $\Gamma$ has bounded torsion so does every element of the deformation. Then the proof of Theorem 5.2 implies the deformation is through isomorphic groups.

We say a negatively curved group is cocompact if the orbit space $\operatorname{int}\left(\mathbf{B}^{n}\right) / \Gamma$ is compact. Notice that cocompact implies nonelementary.

5.5. TheOREM. Let $\left\{\Gamma_{t}: t \in \mathbb{R}\right\}$ be a continuous deformation of a torsion free group $\Gamma_{0}$. If each $\Gamma_{t}$ is a discrete cocompact negatively curved group, then there is a continuous family of homeomorphisms $f_{t}: \mathbf{B}^{n} \rightarrow \mathbf{B}^{n}$ such that $f_{t} \Gamma_{0} f_{t}^{-1}=\Gamma_{t}$. That is, the deformation is topologically trivial.

Sketch of Proof. For each $t$ the quotient is a compact negatively pinched manifold, each with the same isomorphic fundamental group $\Gamma$. The obvious action of this group on the space $\operatorname{int}\left(\mathbf{B}^{n}\right) \times[0,1]$ (here $[0,1]$ is the parameter space) is proper. The orbit space is a manifold foliated by the codimension 1, two-sided compact manifolds (leaves) $\operatorname{int}\left(\mathbf{B}^{n}\right) / \Gamma_{t}$. By Reeb stability, all the leaves are homeomorphic and the orbit space is a trivial fibration. Thus there is a conjugacy $f_{t}: \operatorname{int}\left(\mathbf{B}^{n}\right) \rightarrow \operatorname{int}\left(\mathbf{B}^{n}\right)$. Since each group is cocompact, it is uniform and therefore contains no parabolics [BGS, Lemma 8.2]. The usual Mostow-Margulis construction, see [Mo] and [T], shows that $f_{t}$ is a 
pseudo-isometry, that is, a continuous map which is a bounded distance from an isometry (the minimal number of fundamental domains between two points is preserved by $f$ as it is automorphic). In particular, the image under $f$ of a geodesic line will be a bounded distance from another geodesic line. (Here the curvature assumptions and cocompactness simplify matters greatly. The details are not trivial, see [Mo] and compare with the $\mathbb{R}$-rank one lattice case there. Alternatively the argument given by Thurston [T] works in this general setting). Such maps as $f$ extend homeomorphically to the boundary via their action on geodesic lines.

We remark that the much deeper results of Farrell and Jones [FJ] also imply that the leaves are homeomorphic (for $n \neq 3,4$ ).

\section{REFERENCES}

[B] B. H. Bowditch Discrete parabolic groups, I.H.E.S. Preprint (1990).

[BK] P. Buser and H. Karcher, Gromov's almost flat manifolds, Astérique, 81 (1981).

[BGS] W. Ballman, M. Gromov and V. Schroeder, Manifolds of nonpositive curvature, Progress in Math., 61 (1985) Birkhäuser.

[EO] P. Eberlin and B. O'Neill, Visibility manifolds, Pacific J. Math., 46 (1973), 45-110.

[FJ] F. T. Farrell and L. E. Jones, compact negatively curved manifolds (of dim $\neq$ 3, 4) are topologically rigid, Proc. Nat. Acad. Sci. U.S.A., 86 No. 10 (1989), 3461-3463.

[GM] F. W. Gehring and G. J. Martin, Discrete quasiconformal groups, Proc. London Math. Soc., (3) 55 (1987), 331-358.

[GM2] _-, Inequalities for Möbius transformations and discrete groups, J. Reine Angew. Math., 418 (1991), 31-76.

[G] M. Gromov, Hyperbolic Groups, Essays in group theory. Edited by S. M. Gersten. Springer Verlag (1987), 75-265.

[H] S. Helgason, Differential Geometry and Symmetric Spaces, Academic Press, (1962).

[J] T. Jørgensen, On discrete groups of Möbius transformations, Amer. J. Math., 98, 3 (1976), 739-749.

[JK] T. Jørgensen and P. Klein, Algebraic convergence of finitely generated Kleinian groups, Quart. J. Math. Oxford (2), 33 (1982), 325-332.

[K] K. Kuratowski, Topology, Academic Press, (1966).

[KP] M. Kapovich and L. Potyagailo, On the absence of Ahlfor's finiteness theorem for Kleinian groups in dimension 3, (to appear) Topology Appls.

[M] G. J. Martin, On discrete Möbius groups in all dimensions, Acta Math., 163 (1989), 253-289.

[MS] G. J. Martin and R. Skora, Group actions of the two sphere, Amer. J. Math., 111 (1989), 387-402.

[Mo] G. D. Mostow, The strong rigidity of locally symmetric spaces, Annals of Math. Studies, 78. Princeton Univ. Press, (1973).

[N] M. H. A. Newman, $A$ theorem on periodic transformations of spaces, Quart. J. Math., (2) (1931), 1-8. 
[P] P. Pansu, Métriques de Carnot-Carathéodory et quasi-isometries des espaces symétrique de rang un, Annals. of Math., 129 (1989), 1-60.

[S] A. Selberg, On discontinuous groups in higher dimensional symmetric spaces, Contributions to function theory, Bombay (1960), 147-164.

[Sc] G. P. Scott, Finitely generated 3-manifold groups are finitely presented, J. London Math. Soc., 6 (1973), 437-440.

[T] W. P. Thurston, The geometry and topology of 3-manifolds, Princeton (1978).

[Tu] P. Tukia, On isomorphisms of geometrically finite Möbius groups, Publ. I.H.E.S., 61 (1985), 171-214.

Received October 11, 1990 and in revised form January 18, 1992.

THE UNIVERSITY OF AUCKLAND

AUCKLAND, New Zealand

E-mail address : martin@mat.auckland.ac.nz

AND

INSTITUTE MITTAG-LEFFLER

AURAVÄGEN 17

DJURSHOLM, SWEDEN 



\section{CONTENTS}

G. D. Anderson, M. K. Vamanamurthy, and M. Vuorinen, Inequalities for quasi-

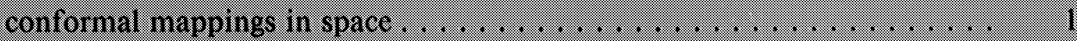

T. Bhattacharya, A nonexistence result for the $n$-Laplacian . . . . . . . . . . . . 19

J. A. Cima, K. Stroethoff, and K. Yale, Bourgain algebras on the unit disk . . . . . 27

J. A. Fridy and C. Orhan, Lacunary statistical convergence . . . . . . . . . . . . . . 43

D. Grenier, On the shape of fundamental domains in $\mathrm{GL}(n, \mathbf{R}) / \mathrm{O}(n) \ldots \ldots . . .53$

B. Jiang and J. Guo, Fixed points of surface diffeomorphisms . . . . . . . . . . . 67

P. Lejarraga, The moduli of rational Weierstrass fibrations over $\mathbf{P}^{\prime}$ : singularities 91

G. J. Martin, On discrete isometry groups of negative curvature . . . . . . . . . . 109

T. Nakashima, Adjoint linear systems on a surface of general type in positive characteristic . . . . . . . . . . . . . . . . . . . . . . . . . 129

B. Ralph, A homotopy transfer for finite group actions $\ldots \ldots \ldots \ldots \ldots \ldots \ldots$

Y. Rong, Maps between Seifert fibered spaces of infinite $\pi_{1} \ldots \ldots \ldots \ldots \ldots, 143$

J.-Y. Shi, Some numeric results on root systems . . . . . . . . . . . . 155

E. Spanier, Singular homology and cohomology with local coefficients and duality

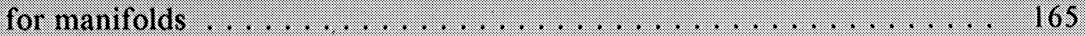




\section{PACIFIC JOURNAL OF MATHEMATICS}

Volume $160 \quad$ No. $1 \quad$ September 1993

Inequalities for quasiconformal mappings in space

GLEN DOUglas ANDERson, MaVina KRISHNa VAMANAMURThy and MATTI VUORINEN

A nonexistence result for the $n$-Laplacian

TILAK BHATTACHARYA

Bourgain algebras on the unit disk

Joseph A. Cima, Karel M. Stroethoff and Keith Yale

Lacunary statistical convergence

JOHN ALBERT FRIDY and CIHAN ORHAN

On the shape of fundamental domains in $\operatorname{GL}(n, \mathbf{R}) / \mathrm{O}(n)$

DOUGLAS MARTIN GRENIER

Fixed points of surface diffeomorphisms

BOJU JIANG and JIANHAN GUO

The moduli of rational Weierstrass fibrations over $\mathbf{P}^{1}$ : singularities

PABLO LEJARRAGA

On discrete isometry groups of negative curvature

GAVEN MARTIN

Adjoint linear systems on a surface of general type in positive characteristic

TOHRU NAKASHIMA

A homotopy transfer for finite group actions

WILLIAM J. RALPH

Maps between Seifert fibered spaces of infinite $\pi_{1}$

YONGWU RONG

Some numeric results on root systems

J. Y. SHI

Singular homology and cohomology with local coefficients and duality for manifolds

EDWIN SPANIER 